\title{
Betrayal Trauma and Gender Differences in Posttraumatic Stress
}

\author{
Sharon Shin Shin Tang and Jennifer J. Freyd \\ University of Oregon
}

\begin{abstract}
The purpose of the current study was to examine the role of betrayal trauma in explaining why women report higher rates of posttraumatic stress than men. Betrayal trauma theory posits that cognitive dissociation is adaptive when trauma occurs at the hands of a caregiver (Freyd, 1996). Betrayal trauma has also been linked to poorer outcomes in mental health, including depression, anxiety, and posttraumatic stress disorder (PTSD) (DePrince \& Freyd, 2002; Goldsmith, 2004). DePrince and Freyd (2002) proposed that the increased frequency with which females experience betrayals accounts in part for the correspondingly higher rates of PTSD and dissociative disorders among women. Thus, it is possible that closeness to the perpetrator acts as a partial mediator of gender differences in prevalence rates (Goldberg $\&$ Freyd, 2006). Using an online survey with a college sample $(n=1,041)$ and a community sample $(n=$ 199), the findings of the current study confirmed prior research that traumas high in betrayal (e.g., abuse by a close other) are more strongly associated with symptoms of posttraumatic stress than traumas lower in betrayal (e.g., natural disaster or abuse by someone not close to the victim). Women also reported higher rates of depression, anxiety, and reexperiencing symptoms of PTSD, but not avoidance and hyperarousal symptoms. The hypothesis that betrayal trauma would mediate the association between gender and PTSD reexperiencing symptoms was supported by statistical significance, but the effect was not substantial.
\end{abstract}

Keywords: betrayal trauma, gender, PTSD

Across socioeconomic status, countries, and cultures, the lifetime prevalence of posttraumatic stress disorder (PTSD) for women is twice that of men. Nationwide studies in the United States have found rates of PTSD to range from $5 \%$ to $6 \%$ in men and $10 \%$ to $13 \%$ among women (Breslau, Chilcoat, Kessler, Peterson, \& Lucia, 1999; Kessler, Sonnega, \& Bromet, 1995). The duration of PTSD is also substantially longer among women, approximately five years in women as compared with 2 years in men (Kessler et al., 1995). The difference in prevalence rates begins to appear around early adolescence when the prevalence of PTSD among females rises more rapidly until it reaches a rate about twice that of males in adulthood (Perkonigg et al., 2000).

Despite the higher prevalence of PTSD among women, men consistently report higher overall rates of exposure to traumatic events (Breslau, 2001; Norris, Foster, \& Weisshaar, 2002), which has led some to suggest an inherent vulnerability among women (Breslau et al., 1997). However, examination of the types of events reveals that men tend to report more instances of accidents, physical assaults, and combat trauma than women, whereas women are more likely to experience sexual assault (Norris et al., 2002). Among all traumas, sexual trauma is associated with one of the highest rates of PTSD. Therefore, the type of trauma may at least partially account for the gender difference in rates of PTSD.

This article was published Online First October 10, 2011.

Sharon Shin Shin Tang and Jennifer J. Freyd, Department of Psychology, University of Oregon.

Correspondence concerning this article should be addressed to Jennifer J. Freyd, Department of Psychology, 1227 University of Oregon, Eugene, OR 97403-1227. E-mail: jjf@uoregon.edu
Tolin and Foa (2006) conducted a meta-analysis of studies of the past 25 years that reported rates of PTSD by gender to investigate whether type of trauma accounts for gender differences in PTSD diagnoses. They found that within types of potentially traumatic events, including natural disasters, accidents, combat, and physical assault, females had higher rates of PTSD and greater severity of PTSD than males. However, child and adult sexual assault were exceptions; no gender differences in rates of PTSD were found for adult sexual assault and males reported slightly greater rates of PTSD than females for child sexual abuse (CSA).

In cases of nonsexual assaultive violence, women have consistently been found to be at increased risk for PTSD (Stein, Walker, \& Forde, 2000; Tolin \& Foa, 2006). Breslau et al. (1999) also found differences in symptom presentation. Avoidance and numbing was more prevalent among females than males exposed to assaultive violence.

A prospective study by Spataro, Mullen, Burgess, Wells, and Moss (2004) examined reported cases of sexual abuse among both boys and girls. As compared with population controls, survivors of CSA exhibited significantly greater rates of anxiety, PTSD, and major affective disorders. Anxiety disorders and PTSD were equally prevalent among male and female survivors; however, they also found that male survivors had a history of more childhood mental disorders than females.

Breslau et al. (1999) examined prevalence rates within four different categories of trauma: assaultive violence, other injuries or shocking events, learning of traumas to others, and the sudden unexpected death of a friend. The study found that the overall gender difference in rates of PTSD was primarily accounted for by exposure to assaultive violence. Breslau (2001) examined the conditional risk for PTSD, or the probability of 
PTSD given a potentially traumatic event. Although the conditional risk for all trauma types was twice as high in women $(13.0 \%)$ than in men $(6.2 \%)$, this difference was primarily due to an elevated conditional risk associated with assaultive violence. In cases of assaultive violence, the conditional risk of PTSD, rose to $35.7 \%$ among women versus $6.0 \%$ in men. Gender differences among other types of trauma (injury or shocking event, learning about trauma to others, and unexpected death of a loved one) were insignificant. Furthermore, over half the cases of PTSD among women were attributable to assaultive violence, as compared with $15 \%$ for men. Kessler et al. (1995) and Neria, Bromet, and Sievers (2002) found that rape was most highly related to PTSD for women, whereas the greatest predictor for men was combat exposure.

Brewin, Andrews, and Valentine (2000) conducted a metaanalysis of risk factors for PTSD among adults and found moderate effect sizes for the type of trauma defined in terms of severity. However, like the Tolin and Foa (2006) findings, gender remained predictive of PTSD even when type of trauma was held constant. Kessler et al. (1995) also found women to be at higher risk for PTSD even when controlling for trauma type.

Gender differences in other anxiety disorders appear to begin much earlier than in PTSD and depression, starting around age 6 when the female to male ratio is already 2:1 (Robinson, 2006). This ratio is consistent across subtypes of anxiety disorders (e.g., generalized anxiety disorder, specific phobias, and obsessivecompulsive disorder) with the exception of social anxiety disorder, in which the lifetime prevalence rate among women is $15.5 \%$ among women and $11.1 \%$ among men (Robinson, 2006).

As with PTSD and anxiety, gender differences in rates of depression begin to emerge in early adolescence (Hankin, Abramson, Moffitt, Silva, \& McGee, 1998; Nolen-Hoeksema, 1990). Between the ages of 15-18, rates of depression among girls rapidly rise to become twice that of the prevalence rate for males (approximately $11 \%$ vs. $23 \%$ ). A longitudinal study of a birth cohort by Hankin et al. (1998) concluded that these differences are entirely due to new cases of depression, rather than the previously hypothesized notion that women experience more recurring cases of depression.

In summary, although the majority of studies have found that women report higher rates of PTSD than men, the type of trauma partially accounts for this difference. The gender difference becomes small to negligible among cases of sexual assault and child abuse. A limitation of some of the studies is that participants were treated as though they had only experienced one type of trauma, without regard to past history. Given that estimated rates of CSA are about $30 \%$ for women and $15 \%$ for men, it is certain that many of the women and men in the various samples experienced CSA in addition to other types of trauma such as car accidents, nonsexual assault, and so forth. Therefore, these results are most likely confounded by experiences of multiple traumas among their participants. Having a history of multiple traumas has been shown to have a more detrimental effect on mental health than single-event traumas. The above studies also did not measure the victimperpetrator relationship that may have accounted for the higher conditional risk among women who experience more assault by close others, that is, the level of betrayal in these traumas.

\section{Betrayal Trauma Theory}

A close, interpersonal relationship to the perpetrator is a distinguishing characteristic of traumas more commonly suffered by females than males. Girls are more likely to be abused by a family member and boys by a nonfamily member (Finkelhor, 1990; Goldberg \& Freyd, 2006). Among victims of interpersonal violence, including sexual assault, women are more likely to be attacked by an acquaintance and men by a stranger. In a community survey, Goldberg and Freyd (2006) found that many more women than men reported having experienced mistreatment by someone close to them, whereas more men than women reported mistreatment by someone not close. Women reported overall higher rates of sexual abuse in both childhood and adulthood. As children, men were 5 times more likely to be physically attacked by someone not close, whereas slightly more women were attacked by someone close. In adulthood, women 6 times more likely than men to be attacked by someone close. Conversely, men were 3 times more likely to be attacked by someone not close.

Research based upon betrayal trauma theory, which distinguishes between traumas perpetrated by a close acquaintance and those that are not, has begun to provide compelling evidence for the consideration of relational influences. Betrayal trauma theory posits that cognitive dissociation is adaptive when trauma occurs at the hands of a caregiver (Freyd, 1996). In support of this theory, Freyd, DePrince, and Zurbriggen (2001) found that close betrayal predicted memory impairment for physical and sexual abuse. Betrayal trauma has also been linked to poorer outcomes in mental health, including depression, anxiety, and PTSD (DePrince \& Freyd, 2002; Goldsmith, 2004). A study of college students by Goldsmith (2004) indicated that gender and betrayal independently predicted anxiety and dissociation scores, supporting the theory that both are important factors to consider.

DePrince and Freyd (2002) proposed that the increased frequency with which females experience betrayals accounts in part for the correspondingly higher rates of PTSD and dissociative disorders among women. Thus, it is possible that closeness to the perpetrator acts as a partial mediator of gender differences in prevalence rates (Goldberg \& Freyd, 2006). Although studies testing this hypothesis with betrayal trauma have not been conducted, those categorizing trauma in a relational context lend support to its plausibility. Among a sample of female sexual assault survivors, Culbertson and Dehle (2001) found more symptoms of hyperarousal among those who were in an acquaintance, married, or cohabiting relationship with their perpetrator, as opposed to those who were dating or sexually intimate with their perpetrator. Women who were acquainted with their perpetrator endorsed more hyperarousal symptoms than those assaulted by a sexually intimate partner whom they were not dating.

Data from the National Comorbidity Study also found that relationship to the perpetrator directly affected outcome in cases of CSA. Sexual assault by step-parents resulted in higher risk of developing PTSD than in cases where the perpetrator was a stranger, even when controlling for chronicity (Molnar et al., 2001). Thus, betrayal trauma may be a particular class of trauma that increases risk of PTSD and other disorders, as compared with traumas of low betrayal. 


\section{Hypothesis 1.1}

HB experiences would be significantly associated with PTSD, depression, anxiety, and dissociation; these associations would also be stronger than those for MB and LB potentially traumatic experiences.

\section{Hypothesis 1.2}

Women would report significantly higher rates of HB experiences than MB and LB experiences. A gender difference between rates of $\mathrm{MB}$ and $\mathrm{LB}$ traumas was not hypothesized.

\section{Hypothesis 1.3}

Women would report higher rates of depression, anxiety, and PTSD than men.

\section{Hypothesis 2}

Experiences of HB trauma would mediate the relationship between gender and PTSD symptoms as shown in Figure 1. Following the steps for conducting a mediation analysis as described by Baron and Kenny (1986), in order for this hypothesis to be supported, several conditions are necessary: 1) Gender must be significantly associated with PTSD, depression, and anxiety; 2) Gender must be significantly associated with HB trauma; 3) HB trauma must be significantly associated with PTSD, depression, and anxiety; 4) the addition of HB trauma to the model results in a significant reduction in the effect of gender on PTSD. The effect of HB trauma on PTSD was depicted as causal as the experience of a traumatic event is a prerequisite for a diagnosis of PTSD, establishing chronicity. Although our instrument was not used for diagnosis, it asked participants to rate their reactions to a traumatic event they had experienced, if any.

Causal paths from trauma to other potential indicators of posttraumatic stress, depression, and anxiety, were not hypothesized due to past findings that indicate that depression and anxiety are not only consequences of trauma, but risk factors as well. Therefore, it is possible that childhood depression or anxiety could have preceded traumatic events. As this study was not longitudinal, it was not possible to distinguish the directional nature of each effect and these variables were not included in the model.

\section{Method}

\section{Participants}

Participants for the study were recruited from the University of Oregon Psychology Department Human Subjects Pool (HSP), as

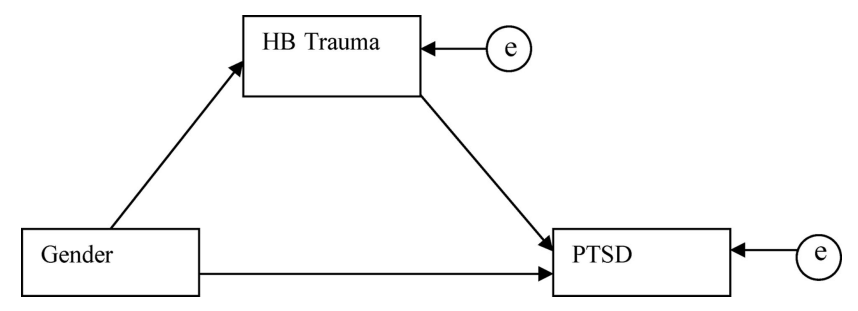

Figure 1. Mediation of gender and PTSD with high-betrayal trauma. well as the general community. Participants did not self-select into the study based on knowledge of the content because they were not informed of the nature of the survey. A total of 1,047 students completed the survey. All were enrolled in introductory psychology or linguistics courses and received course credit for their participation. Six participants were excluded due to apparently invalid responses, namely entering the same response for all items of questionnaires. The remaining 1,041 participants included 705 women and 336 men whose ages ranged from 16 to 54 years with $87.6 \%$ of the sample between the ages of 18 and 21 .

In order to obtain a more diverse sample, participants were also recruited from the general population which comprised adults from the community as well as various universities. Each community participant was paid with a $\$ 10$ gift certificate. Participants in the community cohort were primarily from Oregon with particular emphasis upon Asians and Asian Americans for purposes of another study. They were recruited through listservs of community organizations, posting flyers in the community, placing a public service bulletin in the Asian Reporter, and advertising on the Portland site of Craigslist.org. Finally, recruitment e-mails were sent directly to faculty, students, and staff at the University of Oregon, Oregon State University, and the University of Hawaii who appeared to have Asian surnames.

A total of 216 responses were obtained for the community survey. Of these, it was determined that 12 were duplicate entries based upon similar internet protocol addresses, as well as similar times at which the survey was begun and completed. In these cases, the first set of responses was retained and the rest deleted. Thus, responses for 199 participants were included in the final analyses. These comprised 70 men and 129 women, ages 18-68 years, most of whom $(86.4 \%)$ were between the ages of 18 and 40 . Table 1 compares some of the demographic information for the HSP and the community samples.

\section{Materials}

The online survey began with a brief demographics questionnaire, followed by several measures that evaluated psychological symptoms of distress. A variety of mental health outcome measures were used as recommended by Horwitz, Widom, McLaughlin, and Raskin (2001), who caution that the impact of trauma may differ among social groups. For example, it is possible that the effects of trauma may differ for males and females and this difference may be more pronounced in symptoms of PTSD versus general anxiety.

\section{Trauma Symptom Checklist - 40}

The Trauma Symptom Checklist-40 (TSC-40; Elliot \& Briere, 1992) is a Likert-scale survey that probes a range of symptoms of posttraumatic stress and comprises six subscales: depression, dissociation, anxiety, sexual abuse trauma, sleep disturbance, and sexual problems. The TSC-40 has been demonstrated to have an overall internal consistency of $\alpha=.90$, and the subscales $.62-.77$ (Elliot \& Briere, 1992). Scores are computed by summing all items corresponding to each subscale and computing an average score.

Multiple imputation was used to replace missing items using NORM software (Schafer, 1999) and reliability estimates using the imputed dataset yielded alpha coefficients of .76 for both depres- 
Table 1

Participant Demographic Characteristics for HSP and Community Samples

\begin{tabular}{lcc}
\hline & HSP & Community \\
\hline Total participants & 1041 & 199 \\
Gender & & \\
$\quad$ Females & $705(67.70 \%)$ & $129(64.84 \%)$ \\
$\quad$ Males & $336(32.30 \%)$ & $70(35.16 \%)$ \\
Ethnicity & & \\
$\quad$ African American/Black & $10(1.0 \%)$ & $2(1.0 \%)$ \\
Arab American & $6(0.6 \%)$ & $2(1.0 \%)$ \\
Asian/Asian American & $107(10.3 \%)$ & $156(78.4 \%)$ \\
European American/White & $821(78.9 \%)$ & $29(14.6 \%)$ \\
Latino & $42(4.0 \%)$ & $2(1.0 \%)$ \\
Native American & $21(2.0 \%)$ & $1(0.5 \%)$ \\
Pacific Islander & $9(0.9 \%)$ & $5(2.5 \%)$ \\
Other & $14(1.3 \%)$ & $1(0.5 \%)$ \\
Education & $2(.2 \%)$ & $2(1.0 \%)$ \\
$\quad$ Less than high school & $737(70.8 \%)$ & $27(13.6 \%)$ \\
High school & $255(24.5 \%)$ & $23(11.6 \%)$ \\
Some college & $45(4.3 \%)$ & $66(33.2 \%)$ \\
College (Bachelor degree) & $2(.2 \%)$ & $81(40.7 \%)$ \\
$\quad$ Graduate school & no data & $M=\$ 25,600$ \\
Income & & \\
\hline
\end{tabular}

Note. HSP $=$ University of Oregon Psychology Department Human Subjects Pool.

sion and anxiety. The similarity between reliability coefficients with imputed data and those with list-wise deletion suggests the absence of a systematic pattern to the missing data and provides confidence that the mean of the original items provides a reasonably reliable measure of depression and anxiety.

\section{Revised Civilian Mississippi Scale of PTSD}

Symptoms of PTSD were measured by the Revised Civilian Mississippi Scale of PTSD (R-CMS; Norris \& Perilla, 1996). The first half of the measure asks about feelings and behavior related to a traumatic event and the second half refers to how the participant is currently feeling. Analyses with the R-CMS were run both with and without imputation of missing cases, resulting in reliability coefficients of .91 in both cases.

\section{Brief Betrayal Trauma Survey}

Trauma history was measured using the Brief Betrayal Trauma Survey (BBTS; Goldberg \& Freyd, 2006), a 24-item self-report inventory of low, medium, and high betrayal trauma experiences in childhood and adulthood. LB experiences include natural disasters and car accidents. A MB item is characterized by sexual, emotional, or physical abuse by someone not close. A HB item refers to the same type of events but perpetrated by someone close. The BBTS has been used in numerous studies and has been found to be of minimal risk to participants (Binder, Cromer, \& Freyd, 2004). It also yields good test-retest reliability of $83 \%$ for childhood items and $75 \%$ for events that occurred during adulthood (Goldberg \& Freyd, 2006).

\section{Security and Confidentiality}

Security and confidentiality were primary concerns in the construction and administration of the survey. To this end, the survey was hosted by the server at the Psychology Department of the University of Oregon. Use of a local server rather than a commercial survey website allowed for complete control of data and security. Access to the administrative interface of the web application as well as the database was controlled by hypertext transfer protocol authentication, requiring a username and password to gain access. All communication with the survey application, both by administrators and by potential respondents, was encrypted via the secure socket layer (SSL) protocol and certified with a registered SSL certificate.

\section{Results}

\section{Hypothesis 1.1}

Zero-order correlations among gender, betrayal trauma, and outcome measures are presented in Table 2 for the entire sample of university and community participants. Due to varying sample sizes among the measures, pair-wise deletion was utilized with all available data. The hypothesis that HB trauma would be significantly associated with outcomes of depression, anxiety, and PTSD was supported. LB and MB trauma were also significantly, but less strongly, associated with symptom outcomes. To determine

Table 2

Correlations Among Gender, Betrayal Trauma, and Symptom Outcome Measures

\begin{tabular}{|c|c|c|c|c|c|c|c|c|c|}
\hline & 2 & 3 & 4 & 5 & 6 & 7 & 8 & 9 & 10 \\
\hline 1. Gender $(n=1,240)$ & -.02 & -.01 & -.05 & $.06^{*}$ & $.20^{* *}$ & $.19^{* * *}$ & $.14^{* *}$ & .01 & .05 \\
\hline 2. Age $(n=1,240)$ & & $.10^{\text {** }}$ & $.10^{\text {** }}$ & $.16^{* *}$ & .01 & $-.08^{* *}$ & $-.10^{* *}$ & -.02 & -.01 \\
\hline 3. Low BT $(n=1,226)$ & & & $.35^{* *}$ & $.36^{* *}$ & $.19^{* *}$ & $.21^{* * *}$ & $.18^{* * *}$ & $.18^{* * *}$ & $.21^{* * *}$ \\
\hline 4. Med BT $(n=1,226)$ & & & & $.54^{* * *}$ & $.19^{* * *}$ & $.18^{* * *}$ & $.22^{* * *}$ & $.22^{* * *}$ & $.17^{* * *}$ \\
\hline 5. High BT $(n=1,224)$ & & & & & $.33^{* *}$ & $.30^{* * *}$ & $.27^{* * *}$ & $.29^{* * *}$ & $.24^{* * *}$ \\
\hline 6. Depression $(n=1,240)$ & & & & & & $.76^{* * *}$ & $.43^{* * *}$ & $.46^{\text {*** }}$ & $.58^{* *}$ \\
\hline 7. Anxiety $(n=1,240)$ & & & & & & & $.42^{* * *}$ & $.43^{\text {*** }}$ & $.47^{\text {*** }}$ \\
\hline 8. PTSD reexperiencing $(n=746)$ & & & & & & & $.87^{* * *}$ & $.90^{* * *}$ & $.79^{* *}$ \\
\hline 9. PTSD avoidance $(n=742)$ & & & & & & & & $.87^{* * *}$ & $.66^{* *}$ \\
\hline 10. PTSD arousal $(n=748)$ & & & & & & & & & $.64^{* *}$ \\
\hline
\end{tabular}

Note. $\mathrm{BT}=$ betrayal trauma; PTSD $=$ posttraumatic stress disorder.

${ }^{*} p<.05$. *** $p<.01$. 
Table 3

Comparisons of Correlations of $H B, M B$, and LB Traumas Using Steiger's Z Statistic

\begin{tabular}{lcc}
\hline & HB and MB trauma & HB and LB trauma \\
\hline Depression $(n=1,240)$ & $5.06^{* * *}$ & $4.54^{* * *}$ \\
Anxiety $(n=1,240)$ & $4.35^{* * *}$ & $2.93^{* * *}$ \\
PTSD $(n=741)$ & $2.89^{* * *}$ & $2.58^{* * *}$ \\
\hline
\end{tabular}

Note. $\mathrm{HB}=$ high betrayal $\mathrm{MB}=$ medium betrayal $; \mathrm{LB}=$ low betrayal; PTSD $=$ posttraumatic stress disorder

${ }^{* * *} p<.01$.

whether there was a significant difference among the strengths of these associations, Steiger's $Z$ test was chosen as a more conservative estimate than Hotelling's $t$ statistic to account for conducting multiple comparisons (Steiger, 1980). In all cases the difference was significant (see Table 3 ) and the full hypothesis that HB trauma would have the strongest association with measures of depression, anxiety, and PTSD was supported. Also as expected, there were significant associations between gender and measures of PTSD, depression, and anxiety.

\section{Hypothesis 1.2}

Table 4 presents the means of BBTS subscale scores by gender for low, medium, and high levels of betrayal for the entire sample, and Figure 2 depicts overall rates of reporting. BBTS scores were calculated by summing the number of items endorsed. The maximum number of HB traumas was six, and the maximum number of $\mathrm{MB}$ and $\mathrm{LB}$ traumas was four. Whereas men and women experienced comparable rates of LB and MB experiences, $39 \%$ of women versus $32 \%$ of men reported at least one HB trauma. A directional (one-tailed) Mann-Whitney $U$ test was used to determine whether this difference was significant due to the skewed responses on the BBTS. The Mann-Whitney $U$ is a nonparametric test that does not assume normality and is more conservative than the $t$ test. Approximately one third of the respondents reported not experiencing any HB trauma. The U statistic was converted into $z$ scores in order to evaluate significance. The gender difference was found to be a significant effect, supporting the hypothesis, although the effect size was very small. Effect sizes were calculated using Cohen's $d$.
Figure 3 presents the frequencies with which men and women in the entire sample reported each type of trauma. Women reported approximately $45 \%$ more sexual abuse by someone close and about a third more sexual abuse by someone not close. Women also experienced about a third more emotional abuse than men. Conversely, men experienced 2.5 times more physical assault by not-close others and witnessed one third more attacks.

\section{Hypothesis 1.3}

Multiple analyses of covariance (MANCOVA) were conducted to compare symptom scores by gender. Data from all participants were used in these analyses. One analysis was run for depression and anxiety, and a separate one for the PTSD subscales as the CMS was not completed by all participants. Age was included as a covariate in both analyses as it was previously found to have some small but significant correlations with anxiety and reexperiencing. Descriptive statistics of the symptom scores as well as comparisons of means by gender are reported in Tables 5 and 6. Results of the MANCOVAs showed that women reported significantly higher rates of depression, $F(1,1237)=48.66, p<.001$, and anxiety, $F(1,1237)=44.56, p<.001$ than men, Wilks' $\lambda=.96$. Age was a significant covariate only for anxiety, Wilks' $\lambda=.98$, $F(1,1237)=6.92, p<.01$. Among the PTSD subscales, women reported higher levels of reexperiencing symptoms than men, .97 , $F(1,736)=12.00, p=.001$, but rates of avoidance and arousal symptoms were similar. The effect size of reexperiencing estimated using partial $\eta^{2}$ was very small. In this analysis, age was a significant covariate only for reexperiencing, $.99, F(1,736)=$ $7.42, p<.01$.

\section{Hypothesis 2}

HB trauma was predicted to mediate the association between gender and PTSD. As the only gender difference in PTSD scores occurred for the reexperiencing subscale, this subscale was used in the proposed model in lieu of the total PTSD score. All available data for the university and community samples were used. In order to address missing data, the analysis was run using an expectation maximization (EM) algorithm, which provides maximum likelihood estimates based upon a probabilistic distribution of the data. The analysis also utilized a bootstrap method of random sampling with replacement, in this case with 5,000 iterations. The main

Table 4

Mean BBTS Scores by Gender

\begin{tabular}{|c|c|c|c|c|c|c|}
\hline & \multicolumn{2}{|c|}{$\begin{array}{l}\text { LB trauma } \\
(n=1,226)\end{array}$} & \multicolumn{2}{|c|}{$\begin{array}{l}\text { MB trauma } \\
(n=1,226)\end{array}$} & \multicolumn{2}{|c|}{$\begin{array}{l}\mathrm{HB} \text { trauma } \\
(n=1,224)\end{array}$} \\
\hline & Mean & $S D$ & Mean & $S D$ & Mean & $S D$ \\
\hline Males & .43 & .83 & .36 & .82 & .66 & 1.23 \\
\hline Females & .41 & .74 & .28 & .63 & .81 & 1.29 \\
\hline Mann-Whitney $U$ & \multicolumn{2}{|c|}{168,639} & \multicolumn{2}{|c|}{167,881} & \multicolumn{2}{|c|}{176,566} \\
\hline$Z$ score & \multicolumn{2}{|c|}{0.51} & \multicolumn{2}{|c|}{0.35} & \multicolumn{2}{|c|}{1.92} \\
\hline One-tailed $p$ & \multicolumn{2}{|c|}{0.31} & \multicolumn{2}{|c|}{0.36} & \multicolumn{2}{|c|}{.03} \\
\hline Cohen's $d$ & \multicolumn{2}{|c|}{.02} & \multicolumn{2}{|c|}{.10} & \multicolumn{2}{|c|}{.12} \\
\hline
\end{tabular}

Note. $\quad$ BBTS $=$ Brief Betrayal Trauma Survey; $\mathrm{LB}=$ low betrayal; $\mathrm{MB}=$ medium betrayal; $\mathrm{HB}=$ high betrayal. 


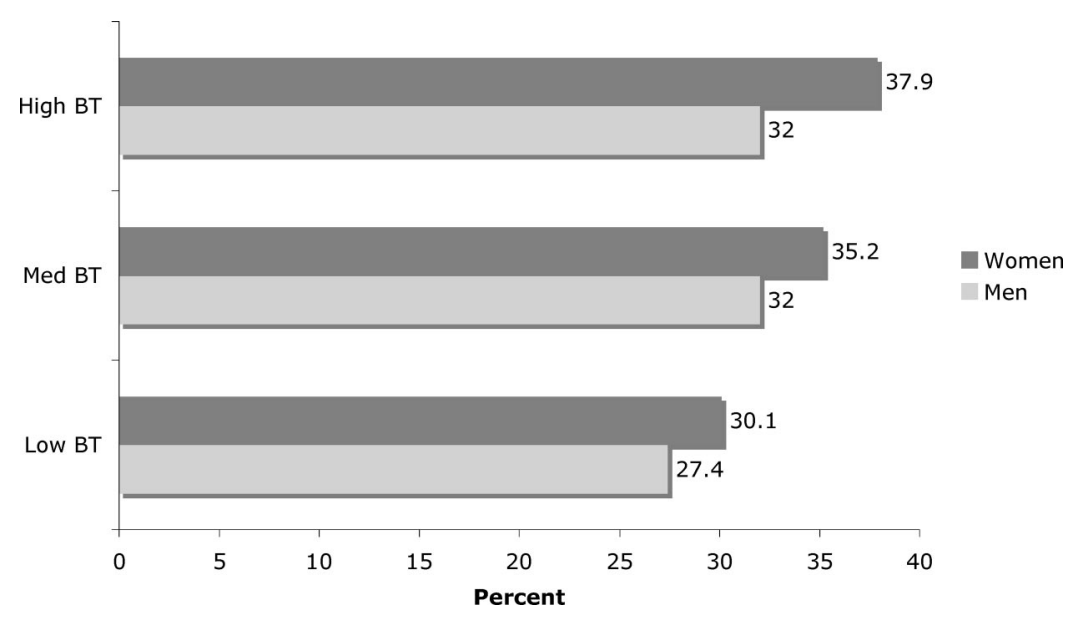

Figure 2. Rates of trauma types reported by gender.

advantage of the bootstrap method is that it does not assume a normal distribution of data, which is useful as the BBTS data were extremely skewed. Significance of the indirect effect of gender on PTSD reexperiencing scores was determined by examining confidence intervals. Those that did not include zero were considered significant. The use of confidence intervals is recommended by MacKinnon (2008) as they provide estimations of the degree of accuracy of an effect in addition to its statistical significance.

Following the steps for mediation by Baron and Kenny (1986), reexperiencing scores were regressed on gender and the relationship was found to be significant. The regression of HB trauma on gender was also significant. Finally, the indirect effect of gender on reexperiencing via $\mathrm{HB}$ trauma was estimated along with the resulting change in association between gender and reexperiencing. Unstandardized parameter estimates are presented in Figure 4, and both standardized and unstandardized estimates in Table 7 . The analysis resulted in a significant effect, as indicated by the $95 \%$ confidence interval falling between .003 to $.05, p<.04$. However, the change in strength of association between gender and reexperiencing from before the mediation (.21) to after the mediation (.20) was very small.

\section{Discussion}

\section{Lifetime Trauma Reports and Gender}

The first objective of the study was to confirm previous findings regarding patterns of association among types of traumatic experiences, psychological sequelae, and gender. As predicted, traumatic events involving a high degree of betrayal were found to be more strongly associated with symptoms of PTSD, depression, and anxiety than were $\mathrm{MB}$ and $\mathrm{LB}$ events. These findings provide additional support for betrayal trauma theory, confirming the increased psychological cost of traumas perpetrated by close others versus those perpetrated by not-close others, or noninterpersonal traumas.

Also consistent with the literature, women reported significantly higher rates of HB trauma than men, particularly sexual assault and emotional abuse. Although the overall effect size was small (Cohen's $d=.12$ ), women reported approximately $45 \%$ more sexual abuse by someone close than men. Women also reported one third more sexual abuse by someone not close. Although a number of studies have found that men typically disclose childhood sexual abuse less often than women, the strength of this trend is unclear. Factors such as age, closeness to the perpetrator, and culture may interact with gender to influence the likelihood of disclosure. (Tang, Freyd, \& Wang, 2007). There was no gender difference for MB and LB traumas although men experienced 2.5 times more physical assault by not-close others and witnessed one third more attacks.

In addition to HB trauma, there was a very small but significant effect of women reporting higher rates of anxiety and depression than men (partial $\eta^{2}=.02$ ). However, contrary to expectations, men and women reported nearly equal rates of overall PTSD symptoms. Women reported higher rates of reexperiencing symptoms but not avoidance and arousal symptoms, and the effect size for reexperiencing was quite small (partial $\eta^{2}=.02$ ). The small effect sizes indicate that the large number of participants contributed to the statistical significance of the findings. In addition, it is difficult to determine whether a larger gender difference in reexperiencing symptoms is typical because few studies of gender differences of PTSD report results by symptom cluster. One study that examined differences in the diagnosis of clinical PTSD was a large national sample conducted in Australia of over 10,000 participants (Peters, Issakidis, Slade, \& Andrew, 2006). They found no difference in the prevalence of the diagnosis of PTSD by standards of the Diagnostic and Statistical Manual for Mental Disorders (4th edition; DSM-IV), but did find that women were more likely to meet both Criterion A (exposure to the traumatic event and strong emotional reaction) and Criterion B of reexperiencing the event.

A study of psychiatric inpatients also found higher rates of reexperiencing symptoms among women than men but not other types of symptoms (Zlotnick et al., 2001). In contrast, other studies suggest that women tend to have more symptoms of arousal and avoidance than men. In a study of motor vehicle accident victims, Fullerton et al. (2001) found no difference in meeting the overall reexperiencing criterion for a diagnosis of PTSD, but did find that women had a higher incidence of reporting specific reexperiencing symptoms, such as being triggered by situation similar to the 


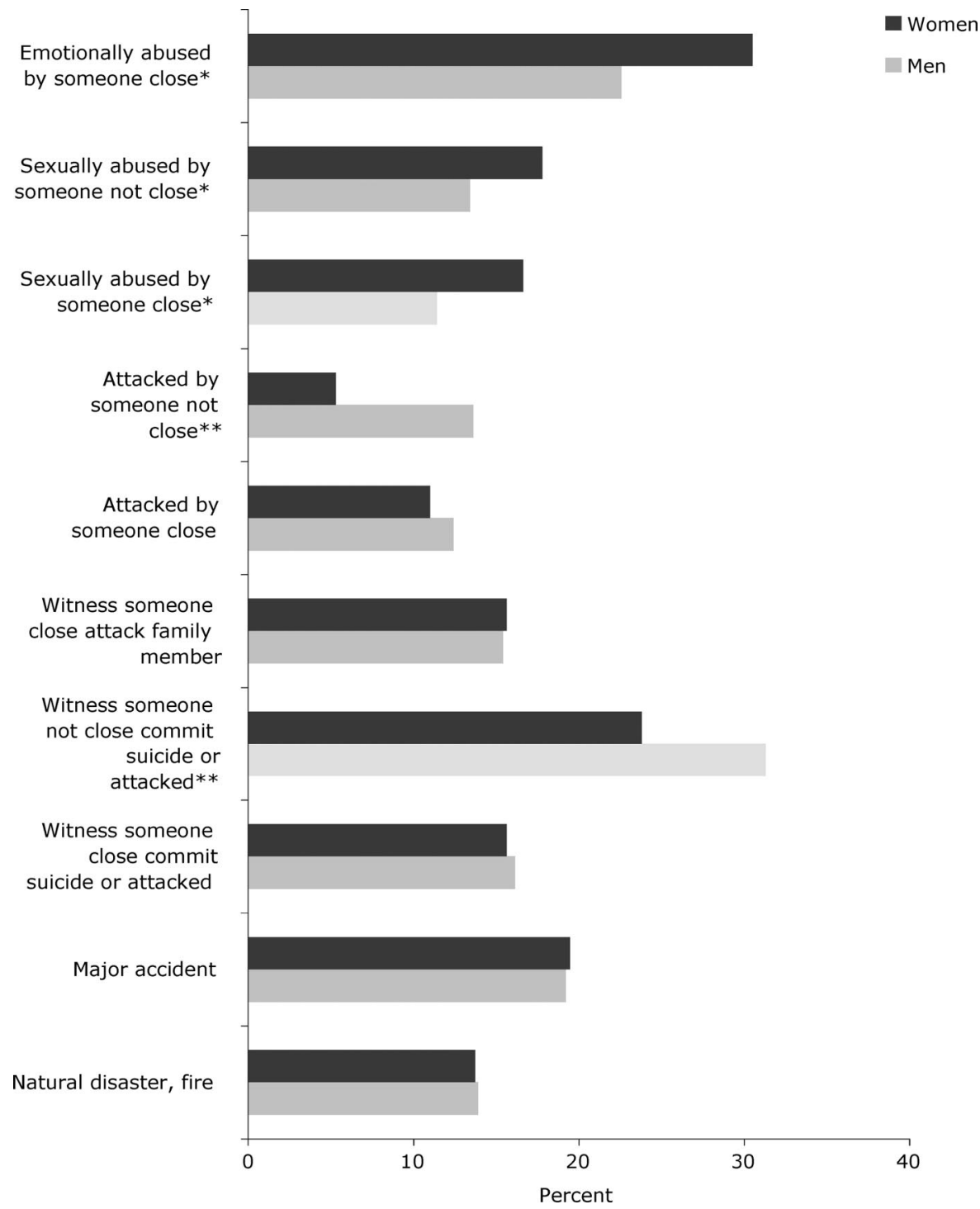

Figure 3. Rates of reported traumas by gender for combined samples.

accident and having distressing memories of the accident, as well as increased symptoms of arousal and avoidance among women.

The type of trauma that occurred may also help explain gender differences among symptoms of PTSD. Breslau et al. (1999) found that assaultive violence was associated with greater gender differences in avoidance and numbing symptoms. Perpetrator type may also influence the impact of sexual assault, as found in a study by Culbertson and Dehle (2001) where closer relationships with the perpetrator were associated with higher levels of hyperarousal symptoms. Additional research is needed in order to understand the potential roles of types of trauma and relationship to the perpetrator may influence PTSD symptom presentation.

Table 5

Comparisons by Gender for the Revised Civilian Mississippi Scale

\begin{tabular}{|c|c|c|c|c|c|c|c|}
\hline \multirow{2}{*}{$\begin{array}{l}\text { Revised Civilian } \\
\text { Mississippi Scale }\end{array}$} & \multicolumn{2}{|c|}{$\begin{array}{c}\text { Male } \\
(n=230)\end{array}$} & \multicolumn{2}{|c|}{$\begin{array}{c}\text { Female } \\
(n=507)\end{array}$} & \multirow[b]{2}{*}{$F$} & \multirow[b]{2}{*}{$p$} & \multirow[b]{2}{*}{ Partial $\eta^{2}$} \\
\hline & Mean & $S D$ & Mean & $S D$ & & & \\
\hline Reexperiencing & 1.82 & .67 & 2.04 & .76 & 12.00 & $<.01$ & .02 \\
\hline Avoidance & 1.92 & .59 & 1.94 & .62 & .07 & .60 & $<.01$ \\
\hline Arousal & 2.28 & .60 & 2.35 & .63 & 1.39 & .24 & $<.01$ \\
\hline
\end{tabular}


Table 6

Comparisons by Gender for Depression and Anxiety Subscales of the TSC-40

\begin{tabular}{cccccccccc}
\hline & \multicolumn{2}{c}{$\begin{array}{c}\text { Male } \\
(n=404)\end{array}$} & & \multicolumn{2}{c}{$\begin{array}{c}\text { Female } \\
(n=818)\end{array}$} \\
\cline { 2 - 3 } $\begin{array}{c}\text { Trauma Symptom } \\
\text { Checklist-40 subscales }\end{array}$ & Mean & $S D$ & & Mean & $S D$ & $F$ & $p$ & Partial $\eta^{2}$ \\
\hline Depression & .61 & .43 & & .81 & .47 & 44.56 & $<.01$ & .04 \\
Anxiety & .66 & .46 & & .87 & .53 & 48.66 & $<.01$ & .04 \\
\hline
\end{tabular}

\section{Gender Differences in Symptoms of PTSD}

The second objective of the study was to investigate why women are diagnosed with PTSD at twice the rate of men, despite reporting fewer overall traumatic events. It was predicted that betrayal trauma would mediate the relationship between gender and PTSD. However, since the only gender difference found was in reexperiencing symptoms, the scores for this subscale were substituted for the scores of the entire PTSD scale. Steps for mediation recommended by Baron and Kenny (1986) were followed, which resulted in a significant mediation effect although the size of the effect was not substantial. The unstandardized coefficient of reexperiencing on gender changed from .21 prior to mediation to .20 . Given the statistical significance but small effect size, further investigation is warranted.

It is possible that an unusually large proportion of men reporting HB trauma in this survey resulted in a weaker association between gender and betrayal trauma and thus a smaller mediation effect. Although a bootstrap technique was used to address the skewed distribution of the BBTS, it is also possible the strength of the association between gender and betrayal trauma was weakened by the high proportion of those who did not report experiencing any traumas (35\%). In addition, although the difference between gender and PTSD reexperiencing symptoms was significant, the effect size was quite small. Finally, the instrument used to measure PTSD, the R-CMS, may have not have been the ideal choice as one study of its psychometric properties indicated that it was more strongly associated with measures of depression and anxiety than other measures of PTSD (Lauterbach, Vrana, King, \& King, 2006).

The methodology of the study may also affect the amount of variance explained in the relationship between gender and PTSD symptoms. Tolin and Foa (2006) found that the method of data collection affected findings of gender differences in traumatic experiences. Studies using surveys such as the current project were less likely to find differences than those conducted with structured interviews. This may occur due to undermeasuring of traumatic

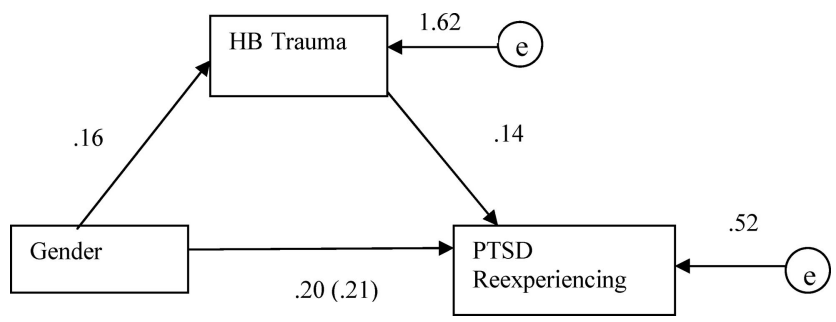

Figure 4. Mediation of gender and PTSD reexperiencing symptoms by HB trauma using EM. events. A meta-analysis by Bolen and Scannapieco (1999) concluded that estimates of abuse are likely to underestimate the true prevalence, especially when based upon a single report. In addition, Tolin and Foa (2006) suggest that samples of college students may underrepresent victims of childhood sexual abuse, the majority of whom are women, as CSA is associated with higher school dropout rates. Further research is needed to replicate the statistical significance of the mediation findings, replicate the effect size, and evaluate whether that small effect size is an artifact of an aspect of this study or actually represents a small effect in the real world. Given the limitations of university samples and survey methodology, it would be ideal to gather data using a randomized sample of community participants with structured interviews.

Another factor that may account for unexplained variance in the mediation model are experiences of assaultive violence that were not categorized as high in betrayal. Although there was no overall gender difference in $\mathrm{MB}$ experiences, women reported significantly more sexual assault by not-close others than men. Breslau et al. (1999) found that assaultive violence accounted for much of the gender difference in diagnoses of PTSD in a community sample, possibly because assaultive violence can be more threatening to women. In the current study, the questionnaires did not link symptoms to a specific trauma event. Therefore, adding sexual assault by not-close others as a mediator may help explain more of the association between gender and PTSD symptoms.

These results establish that further investigation of these social contextual factors is warranted in deepening our understanding of the causes of gender differences in posttraumatic stress. The findings have important clinical implications for diagnosing PTSD and other trauma-related disorders. They support the argument of Brown and Freyd (2008) that Criterion A, which defines the characteristics of a traumatic event, should be expanded to include betrayal in addition to traditional conceptualization of fear and horror. Doing so would allow nonviolently exploited individuals to qualify for a diagnosis of PTSD who would otherwise be excluded even if they met the symptom criteria.

Understanding the etiology of PTSD can also help improve treatment selection and efficacy. For example, someone who ex-

Table 7

Mediation Model Estimates

\begin{tabular}{llll}
\hline & $B$ & $S E$ B & $\beta$ \\
\hline Reexperiencing on & & & \\
HB Trauma & .14 & .02 & .24 \\
$\quad$ Gender & .20 & .06 & .12 \\
HB Trauma on Gender & .16 & .08 & .06 \\
\hline
\end{tabular}


perienced abuse by someone close may require therapy focusing upon repairing interpersonal skills using relational therapy more so than someone who experienced a lower betrayal trauma such as an automobile accident. In addition, if it is true that having more egalitarian gender role beliefs can improve outcomes among men, then perhaps these can be encouraged in a clinical setting as well.

\section{References}

American Psychiatric Association. (2000). Diagnostic and statistical manual of mental disorders: DSM-IV-TR. Washington, DC: Author.

Baron, R. M., \& Kenny, D. A. (1986). The moderator-mediator variable distinction in social psychological research: Conceptual, strategic and statistical considerations. Journal of Personality and Social Psychology, 51, 1173-1182. doi:10.1037/0022-3514.51.6.1173

Binder, A., Cromer, L. D., \& Freyd, J. J. (2004, November). What's the harm in asking? Participant reaction to trauma history questions compared with other personal questions. Poster presented at the 20th Annual Meeting of the International Society for Traumatic Stress Studies, New Orleans, LA.

Bolen, R. M., \& Scannapieco, M. (1999). Prevalence of child sexual abuse: A corrective metanalysis. Social Service Review, 73, 281-313. doi: $10.1086 / 514425$

Breslau, N. (2001). The epidemiology of posttraumatic stress disorder: What is the extent of the problem? Journal of Clinical Psychiatry, 62, $16-22$.

Breslau, N., Chilcoat, H. D., Kessler, R., Peterson, E. L., \& Lucia, V. C. (1999). Vulnerability to assaultive violence: Further specification of the gender difference in posttraumatic stress disorder. Psychological Medicine, 29, 813-821. doi:10.1017/S0033291799008612

Breslau, N., Davis, G. C., Peterson, E. L., \& Schultz, L. (1997). Psychiatric sequelae of posttraumatic stress disorder in women. Archives of General Psychiatry, 54, 81-87.

Brewin, C. B., Andrews, B., \& Valentine, J. D. (2000). Meta-analysis of risk factors for posttraumatic stress disorder in trauma-exposed adults. Journal of Consulting and Clinical Psychology, 68, 748-766. doi: 10.1037/0022-006X.68.5.748

Brown, L. S., \& Freyd, J. J. (2008). PTSD criterion A and betrayal trauma: A modest proposal for a new look at what constitutes danger to self. Trauma Psychology, Division 56, American Psychological Association, Newsletter, 3, 11-15.

Culbertson, K. A., \& Dehle, C. (2001). Impact of Sexual Assault as a Function of Perpetrator Type. Journal of Interpersonal Violence, 16, 992-1007. doi:10.1177/088626001016010002

DePrince, A. P., \& Freyd, J. J. (2002). The intersection of gender and betrayal in trauma. In R. Kimerling, P. C. Ouimette, \& J. Wolfe (Eds.), Gender and PTSD (pp. 98-113). New York, NY: Guilford Press.

Elliot, D. M., \& Briere, J. (1992). Sexual abuse trauma among professional women: Validating the Trauma Symptom Checklist - 40 (TSC-40). Child Abuse \& Neglect, 16, 391-398. doi:10.1016/0145-2134(92) 90048-V

Finkelhor, D. (1990). Early and long-term effects of child sexual abuse: An update. Professional Psychology, 21, 325-330. doi:10.1037/07357028.21.5.325

Freyd, J. J. (1996). Betrayal trauma: The logic behind forgetting childhood abuse. Cambridge, MA: Harvard University Press.

Freyd, J. J., DePrince, A. P., \& Zurbriggen, E. L. (2001). Self-reported memory for abuse depends upon victim-perpetrator relationship. Journal of Trauma \& Dissociation, 2, 5-17. doi:10.1300/J229v02n03_02

Fullerton, C. S., Ursano, R. J., Epstein, R. S., Crowley, B., Vance, K., Kao, T., . . Baum, A. (2001). Sex differences in posttraumatic stress disorder after motor vehicle accidents. American Journal of Psychiatry, 158, $1468-1491$

Goldberg, L. R., \& Freyd, J. J. (2006). Self-reports of potentially disturbing experiences in an adult community sample: Gender differences in event frequencies, test-retest stabilities, and the hierarchical factor structure of the items in a Brief Betrayal Trauma Survey. Journal of Trauma \& Dissociation, 7, 39-63. doi:10.1300/J229v07n03_04

Goldsmith, R. E. (2004). Physical and emotional health effects of betrayal trauma: A longitudinal study of young adults. (Doctoral dissertation, University of Oregon). Retrieved from http://dynamic.uoregon.edu/ jjf/ theses/goldsmith04.pdf

Hankin, B. L., Abramson, L. Y. Moffitt, T. M., Silva, P. A., \& McGee, R. (1998). Development of depression from preadolescence to young adulthood: Emerging gender differences in a 10-year longitudinal study. Journal of Abnormal Psychology, 107, 128-140. doi:10.1037/0021843X.107.1.128

Horwitz, A. V., Widom, C. S., McLaughlin, J., \& White, H. R. (2001). The impact of childhood abuse and neglect on adult mental health: A prospective study. Journal of Health and Social Behavior, 42, 184-201. doi: $10.2307 / 3090177$

Kessler, R. C., Sonnega, A., \& Bromet, E. (1995). Posttraumatic stress disorder in the national comorbidity survey. Archives of General Psychiatry, 52, 1048-1060.

Lauterbach, D., Vrana, S., King, D. W., \& King, L. A. (2006). Psychometric properties of the Civilian Version of the Mississippi PTSD scale. Journal of Traumatic Stress, 10, 499-513. doi:10.1002/jts.2490100313

MacKinnon, D. P. (2008). Introduction to statistical mediation analysis. Mahwah, NJ: Erlbaum.

Molnar, B. E., Buka, S. L., \& Kessler, R. C. (2001). Child sexual abuse and subsequent psychopathology: Results from the National Comorbidity Survey. American Journal of Public Health, 91, 753-776. doi:10.2105/ AJPH.91.5.753

Neria, Y., Bromet, E. J., \& Sievers, S. (2002). Trauma exposure and posttraumatic stress disorder in psychosis: Findings from a firstadmission cohort. Journal of Counseling and Clinical Psychology, 70, $246-251$.

Nolen-Hoeksema, S. (1990). Gender differences in depression. Stanford, CA: Stanford University Press.

Norris, F., \& Perilla, J. (1996). The Revised Civilian Mississippi Scale for PTSD: Reliability, validity, and cross-language stability. Journal of Traumatic Stress, 7, 447-456.

Norris, F. H., Foster, J. D., \& Weisshaar, D. L. (2002). The epidemiology of gender differences in PTSD across developmental, societal, and research contexts. In R. Kimerling, P. C. Ouimette, \& J. Wolfe (Eds.), Gender and PTSD (pp. 3-42). New York, NY: Guilford Press.

Perkonigg, A., Kessler, R. C., Storz, S., \& Wittchen, H.-U. (2000). Traumatic events and post-traumatic stress disorder in the community: Prevalence, risk factors and comorbidity. Acta Psychiatrica Scandinavica, $101,46-59$

Peters, L., Issakidis, C., Slade, T., \& Andrews, G. (2006). Gender differences in the prevalence of DSM-IV and ICD-10 PTSD. Psychological Medicine, 36, 81-89. doi:10.1017/S003329170500591X

Robinson, G. E. (2006). Gender differences in depression and anxiety disorders. In S. E. Romans \& M. V. Seeman (Eds.), Women's mental health. New York, NY: Lippincott, Williams, \& Wilkins.

Schafer, J. L. (1999). Multiple imputation: A primer. Statistical Methods in Medical Research, 8, 3-15. doi:10.1191/096228099671525676

Spataro, J., Mullen, P. E., Burgess, P. M., Wells, D. L., \& Moss, S. A (2004). Impact of child sexual abuse on mental health: Prospective study in males and females. The British Journal of Psychiatry, 184, 416-421. doi:10.1192/bjp.184.5.416

Steiger, J. H. (1980). Tests for comparing elements of a correlation matrix. Psychological Bulletin, 87, 245-251.

Stein, M. B., Walker, J. R., \& Forde, D. R. (2000). Gender differences in susceptibility to posttraumatic stress disorder. Behavior Research and Therapy, 38, 619-628. doi:10.1016/S0005-7967(99)00098-4

Tang, S. S., Freyd, J. J., \& Wang, M. (2007). What do we know about 
gender in the disclosure of child sexual abuse? Journal of Psychological Trauma, 6, 1-26. doi:10.1080/19322880802096442

Tolin, D. F., \& Foa, E. B. (2006). Sex differences in trauma and posttraumatic stress disorder: A quantitative review of 25 years of research. Psychological Bulletin, 132, 959-992. doi:10.1037/0033-2909.132.6.959

Zlotnick, C., Shea, M. T., Begin, A., Pearlstein, T., Simpson, E., \& Costello, E. (1996). The validation of the Trauma Symptom
Checklist-40 (TSC-40) in a sample of inpatients. Child Abuse and Neglect, 20, 503-510.

Received May 25, 2010

Revision received January 24, 2011

Accepted February 7, 2011

\section{Members of Underrepresented Groups: Reviewers for Journal Manuscripts Wanted}

If you are interested in reviewing manuscripts for APA journals, the APA Publications and Communications Board would like to invite your participation. Manuscript reviewers are vital to the publications process. As a reviewer, you would gain valuable experience in publishing. The P\&C Board is particularly interested in encouraging members of underrepresented groups to participate more in this process.

If you are interested in reviewing manuscripts, please write APA Journals at Reviewers@apa.org. Please note the following important points:

- To be selected as a reviewer, you must have published articles in peer-reviewed journals. The experience of publishing provides a reviewer with the basis for preparing a thorough, objective review.

- To be selected, it is critical to be a regular reader of the five to six empirical journals that are most central to the area or journal for which you would like to review. Current knowledge of recently published research provides a reviewer with the knowledge base to evaluate a new submission within the context of existing research.

- To select the appropriate reviewers for each manuscript, the editor needs detailed information. Please include with your letter your vita. In the letter, please identify which APA journal(s) you are interested in, and describe your area of expertise. Be as specific as possible. For example, "social psychology" is not sufficient-you would need to specify "social cognition" or "attitude change" as well.

- Reviewing a manuscript takes time (1-4 hours per manuscript reviewed). If you are selected to review a manuscript, be prepared to invest the necessary time to evaluate the manuscript thoroughly. 